CASOS CLINICOS

Rev. Chil. Pediatr, 67 (2); 75-78, 1996

\title{
Macroprolactinoma en adolescentes: respuesta al tratamiento con bromocriptina
}

\author{
María Loreto Reyes G. '; Claudia Campusano M. ${ }^{2}$; Andreína Cattani O. ${ }^{2}$
}

\section{Macroprolactinomas in children. Bromocriptine treatment}

\begin{abstract}
Maciopiolactinomos appea to oe rare in children and adolescents Ir adults, bromocriptine therapy has shown successtu esults. Three adolescant polients with mocroprolactinomo, their cl'nical courses ond response to bromocriptine treclment are reportec. The patients were wo girls, aged 14 and 17.6 years, with ame-orihea. galactorrhea and headache and ar 18 year old boy with delayed growth ano puberky. hypothyroid sm and growth homone deficiency. All of them had vyperprolactinemia and a pituitary ur ar larger than 10 mm. After bromocriptine realmen roloctinemio became nermal and the tumar disopoeared in two palients. One of the offected girls showed on'y partial response In accordance with these resuits and those reported in the literature, bromocrionline seems to be an effective thercpy, even in cases with visual feid cetects or hypopituitarism, and. as in adult patients, it should also ve considered the therapy of choice for macroprolactiroma in ado escents
\end{abstract}

KKey words: hyperpolactinemia, arclactinom, bromocriptine.l

Los macroprolactinomas son raros en niños $y$ adolescentes. Se han publicados aproximadamente treinta casos en menores de 18 años, la mayoría de ellos han sido tratados quirúrgicamente ${ }^{1-4}$. En adultos, la terapia exclusiva con bromocriptina ha desplazado a la cirugía como primera eleccion ${ }^{5-7}$.

En los últimos 4 años henos tenido la oportunidad de identificar tres casos de macroprolactinomas en adolescentes cuya forma de presentación, evolución clínica y respuesta al tratamiento con bromocriptina se describen a continuación con el propósito de dar mayor difusión a] problema.

1. Becada. Departamentos de Pediatria y Endocrinología, Metaholismo y Nutrición. Facultad de Medicina. Ponlificia Liniversidad Católica de Chile.

2. Departamentos de Pedialria y Endocrinología, Metabolismo y Nutrición, Faculad de Medicina. Pontificia Universidad Católica de Chile

Presentado en la VIII Reunión Anual de Ia Sociedad Latinaamericana de Endocrinología Pediátrica (SLEP), octubre 1944.

\section{Casos clínicos}

1. Niña que consultó a los $\mathbf{1 4}$ años por amenorrea secundaria de 6 mescs de evolución. Había presentado su menatquia a los 11 años a la que siguieron menstcuaciones regulares por 2 affos. Referfa, como síntoma asociado. cefalen bitemporal pulsátil. Su talla era de $156 \mathrm{~cm}$ (p 25 NCHS), indice peso/talla: 105\%, desartollo mamario $y$ veIto púbico en etapa IV de Tanner. Gadactorrea bilateral. Prolactinemia: $175 \mathrm{ng} / \mathrm{ml}$ ( VN $<20$ ); TSH: $2,9 \mu \mathrm{U} / \mathrm{ml}$ ( VN 0,5 - 5): $T_{4}: 6,2 \mu \mathrm{g} / \mathrm{dl}$ (VN 6,5 - 12,5); FSH: $14,9 \mathrm{mU} / \mathrm{ml}$ (VN $[-20$ ). En la tomografía axial computadorizada cerebral se registró un tumor intraselar de $24 \mathrm{~mm}$ de diánetro. que invadía el seno esfenoidal. La campimetría optica de Goldman era nomal, sin delerioro visual. Se índico uatamiento con bromocriptina en dosis crecientes hasta 7.5 $\mathrm{mg}$ - día, que fueron bien toleradas; la prolactinemia se Dormalizó un mes después de comenzar este tratamiento y las menstruaciones reaparecieron al cabo de cuatro tneses, momento en el cual en la tomografía axial había desaparido el tumor y se había reconstituido el techo esfe. noidal. La dosis del medicamento fue disminuida a $5 \mathrm{mg}$ • día y al cabo de 2 años de segumiento, la prolactinemia y la tomografia axial selar son normales y sus menstruaciones son regulares.

2. Niña de 17 años y 8 meses que consultó por ame. norrea primarin, habiendo iniciado relarquia y pubarquia aproximadamente un año antes. Visión borrosa y cefalea holocranea de un año de evolución. Su talla era de 154,6 em (pl0 NCHS); el índice peso/talla 139\%; la etapa de desarrollo (Tanner) de mamas y vello púbico era IIf y II 
respcctivaneote. La concentración de profactina sérica era $790 \mathrm{ng} / \mathrm{ml}$ : TSH 2.3 $\mu \mathrm{L} / \mathrm{m}$ ]; $\mathrm{T}_{4} 9.8 \mu \mathrm{g} / \mathrm{dl}:$ FSH $3,9 \mathrm{~mL} / \mathrm{ml}$; LH de $4.5 \mathrm{mU} / \mathrm{ml} ; \mathrm{E}_{-} 49 \mathrm{rg} / \mathrm{ml}$. Mediante tomografía axial se dercctó un tumor intraselar y supraselar de 13 mon de diánetro con erosión del piso de la silla turca y de la apófisis clinoides anterior izquieruba y leve desplazamiento superior del quiasina óptico. que se acompañaba de hemianopsia bitemporal. Fue tratada con bromocriptina en dosis pogresivas hasta $25 \mathrm{mg}$ - día durante un año. logríndose reducción de la concentración de prolactina sérica a 146 $n g / m l$ disminución de $60 \%$ del tamaño del tumor $y$ normalización del campo visual. Sin embargo ha persistido la amenortea y no ha habido progreso en los eventos puberales. Actualmeste completó cuatro meses de tratamiento con otro unálogo dopaminérgico (CV 205-502), sin que se obsirven cambios en el lamaño del tumor o cambios susianciales con respecto a los resultados obtenidos con bromocriptisa pot lo que se ha planteado la resección quirúrgica como la siguente opción terapúutica.

3. Varon de 18 anos de edad, que consulto por retardo de] crecimiento y del desafrollo puberal. Además sufría con frecuencia cefalea holocránea. Su taila erá $160 \mathrm{~cm}$ ip < 3 OMS): índice peso/tala lo8c: aumento de la graso del troco: desarcollo genital en etapa III de Tanner. Prolactina súricu ISIE $\mathrm{ng} / \mathrm{ml}$ : TSH $2.3 \mu \mathrm{g} / \mathrm{dl}$; prueba de estímulo para hormona de crecimicnto (GH) con clonidina (previa scnsibilización con estradiol), con respues1a máxima de $\mathbf{G H}$ (le $0.8 \mathrm{ng} / \mathrm{m}$ ! (VN > 7). Edad ósea (Greulich y Pyle) 15 aìos 3 meses. En la lomografía axial se registró un lunor intaselar de 19 mo de diámetro. No había alteraciones del campo visual.

Se indicó sustitución con hormona tiroidea y bromocripina eu dosis progresivas hasta $7,5 \mathrm{mg}$ • día, con buena 1olerancia. Dos mescs después, la prolactinemia y la respuesia de la hormona de crecimiento a clonidina cran normales. En lit tomografia axjal realizada cinco meses desputs de comenzas el tratamienlo no se encontraron signos del 1umor. Durante el primer afto de trataniento crectó J tr cin y compleló su desartollo puberal. Actualmente, al cabo de dos años de ratamicnto, recibe $5 \mathrm{mg} \cdot$ dia de hromocriptina y las concentraciones séricas de prolactiua y testosterona se mastienen normales.

\section{Comentario}

Los macroprolactimomas o adenomas tactotropos mayores de $10 \mathrm{~mm}$ son infrecuentes en pacientes menores de 18 años. Entre ellos, los adolescentes son los más afectados. El pacicnte más joven descrito ha sido un niño de 6 $\operatorname{añ} 0 s^{89}$

Las manifestaciones clínicas de estos tumores se deben a la hiperprolactinemia por sí misma y al déficil de otras hormonas hipofisiarias, cuya causa puede ser funcional o anatónica, por el efecto de masa sobre las estructuras adyacentes. La hiperprolactinemia inhibe la secreción pulsátil de hormona liberadora de gonadotrofinas. lo que inicialmente altera la producción cíclica de ellas y conduce luego a hipogonadismo hipogonadotrópico ${ }^{10}$. El déficit de producción de otras hormonas adenohipofisiarias es frecuente, siendo el más comín el de hormona de crecimiento (9 de 11 casos), seguido por el de gonadotrofinas ( 6 de 10 casos) y, en menor proporción, los de TSH y ACTH (4 y 3 de 11 casos respectivamente) $)^{2,4,7.9 .15,36 . ~ E n t r e ~ l a s ~}$ manifestaciones secundarias al crecimiento del tumor. la cefalea se observa, como en los adultos. en aproximadamente la mitad de los casos. El defecto del campo visual y los síntomas de hipertensión endocraneana son menos frecuentes $2.3 .4,10.15$.

La forma de presentación clínica más frecucntc en las niñas es la amenorrea con galactorrea, asociadas a retardo del desarrollo puberal. En los varones, las manifestaciones más comunes son los retardos del crecimiento $y$ del desarrollo puberal $2-4,9,11-14$.

A diferencia de lo sugerido en las primeras publicaciones sobre esta enfermedad, que sugerían que los macroprolactinomas eran más agresivos en los niños, en experiencias más recientes éstos muestran comportamiento semejante al del adulto en su presentación, la respuesta al tratamiento y el pronóstico $4.9 .14,17-19$. To que ha licvado a cambiar la aproximación terapéutica ante los macroprolactinomas de los niños, adoptando una conducta más conservadora.

La cirugía transesfenoidal en los pacientes adultos produce resultados poco satisfactorios, con $40 \%$ a $80 \%$ de recidivas a los 5 años de seguimiento ${ }^{5,10}$. De los 7 casos en niños sometidos a cirugía, 5 recidivaron en los 3 años siguientes; en otra serie de 18 pacientes con prolactinomas $67 \%$ requirieron Iratamiento médico o radioterapia después de la cirugía para controlar la enfermedad ${ }^{l}$.

Por otra parte, en adultos, el manejo de macroprolactinomas con radioterapia como único tratamiento sólo excepcionalmente se asocia con recidiva tumoral, pero el efecto benéfico tarda meses o años en ocurrir y con mucha frecuencia se produce daño de la hipófisis con déficil de hormona de crecimiento en todos los casos al cabo de dos años de seguimiento, de gonadotropinas en $50 \% 10$ años después y de ACTH y TSH en cerca de $14 \%^{5}, 10$. Esta alta incidencia de hipopituitarismo después de la radioterapia la convierten en una indicación excepcional en niños, en quienes se reserva sólo 
para los macroprolaclinomas invasores que no responden a medicamentos dopaminérgicos o cirugía.

La bromocriptina. introducida en 1971, ha sido empleada en el tralamiento de hiperprolactinemias de diversas causas. Es un agonista dopaminérgico derivado del cornczuelo del centeno (ergot) que inhibe la síntesis y secreción de prolactina en lactotropos normales y tumorales. La reducción del tamaño del tumor que produce sc debe a disminución del volumen celular, inhibición de la proliferación y, en menor grado, destrucción de células (necrosis). Si bien produce rápida y notoria reducción del tamaño de los tumores, éstos pueden volver a crecer al suspenderla ${ }^{7} .21 .22$. Más de $80 \%$ de los macroprolactinomas en adultos responden a la bromocriptina, que es considerada el medicamento de primera elección para su tratamiento, aun en tumores grandes con compromiso de campo visual e hipopituitarismo ${ }^{5.10}$. El fármaco puede producir electos secundarios como mareos, náuseas, vómitos e hipotensiónn postural, que pueden ser evitados administrándola en dosis progresivamente crecientes ${ }^{\overline{ }}$. En niños se han comunicado 1) casos mánejados con ella como tratamiento inicial. 10 respondieron satisfactoriamente y no requirieron otra terapia.4. \%. 14, 17-19, en todos el Inedicamento fue bien tolerado. Como en los adultos. la bromocriptina debe ser administrada por liempos prolongados en los niños afectados, pero no se ha podido definir aún la duración ideal del tratamiento $0^{6.7 .18}$. El uso prolongado del medicamento no parece afectar ol creciinienlo y desarrollo del adolescente y tampoco contraindicar la posibilidad de una futura gestación. La bromocriptina no ha mostrado efectos teratogénicos en animales o en humanos, por 10 que se recomienda mantenerla durante el emharayo en mujeres con macroprolactinomas, para disminuir el riesgo de reexpansión tumoral. especialmente si se ha completado menos de un año de tratamiento ${ }^{22}$. En la mayoría de los pacientes con mactoprolactinomas, la prolactinemia se hace normal con dosis entre $7,5 \mathrm{y}$ $15 \mathrm{mg}$ - día. La dosis máxima no ha sido bien determinada y algunos adultos han recibido hasta $60 \mathrm{mg}$ - día. Algunos han definido la resistencia a la bromocriptina como la falla en conseguir prolactinemia normal en tres a seis meses con dosis mayores de $15 \mathrm{mg} \cdot$ día $^{23}{ }^{24}$. Los asonistás dopaminérgicos de acción prolongada como -por ejemplo- pergolide, no son más efectivos que la bromocriptina en estos casos: su única ventaja sobre ella sería que, por su vida media más larga, se pueden usar en una dosis diaria, facilitando la adhesion al tratamiento ${ }^{25}$. Recientemente ha sido desarrollado un nuevo análogo dopaminérgico no derivado del ergot, llamado CV 205-502 o quinagolide, que ha sido probado en adultos con macroprolactinomas resistentes a bromocriptina, con resultados promisorios $^{26-28}$.

De los casos que comentamos dos pacientes tuvieran excelente rcspuesta a la bromocriptina con recupcración de la función gonadal y de la de hormona de crecimiento en uno de ellos. Estos hechos pueden ser explicados por la corrección del hipotiroidismo y reducción del efecto de masa del tumor sobre los somatotropos. En la otra paciente, si bien la respuesta fue parcial, ésta permitió revertir el compromiso visual y convertir un macrotumor en un microtumor hipofisiario, lo que facilitará el abordaje quirúrgico y disminuirá el riesgo de complicaciones asociadas a la operación. Esta y otras experiencias sugieren que la bromocriptina es un medicamento seguro $y$ efectivo para el manejo de macroprolactinomas en adolescentes y debe ser considerada en primer témino en ellos.

\section{Resumen}

Los macroprolactinomas son raros en niños $y$ adolescentes. En adultos el tratamiento con bromocriptina (BC) ha sido exitoso. Existe escasa información en relación a su uso en edad pediátrica. Sc describen tres casos de adolescentes con macroprolactinoma, tratados con bromocriptina, dos mujeres de 14 y 17,6 años con amenorrea, galactorrea, cefalea y un varón de 18 años con retardo del crecimiento y el desarrollo puberal, hipotiroidismo y déficit de hormona de crecimiento. Todos tenían hiperprolactinemia (> $150 \mathrm{ng} / \mathrm{ml}$ ) y tumor selar mayor de $10 \mathrm{~mm}$. Después de tratamiento con bromocriptina se consiguió en dos de ellos prolactinemia normal y desaparición completa del tumor. Una de las niñas rspondió sólo parcialmente al tratamiento. La bromocriptina es pues un tratamiento efectivo, aun en pacientes con deterioro del campo visual e hipopituitarismo $y$, como en adultos, debería ser considerada el 
tratamiento de primera elección en adolescentes con macroprolactinomas.

(Palabras clave: hiperprolactinemia, prolactinoma, bromocriptina.)

\section{Referencias}

I. Dyer F. Cinit $T$, Visat A, Delalande O. Derome P: Transphenoidal surgery for pituitary adenomas in children. Neurosurgery 1994; 34: 207-212.

2. Billated L. Cousin I. Guilhaume B. Lutof JP. Prolactin adenoma developed during puberty. Diagnosis and long1erin course, 9 cases. Presse Med 1993: 22: 299-303.

3. Huddet $S$, Vil Gilder 1. Menezes A: Pediatric piluilary tumors. Neurosurgery 1991: 29:509.514

4. Tricery $C$ : Prolactinoma in an adolescent girl: unusual response to bromocriptine therapy. An $J$ Dis Child 1989: 14.3: 521 - 523 .

5. Besser $M$ : Criteria for medical as opposed to surgical treatment of prolactinomas. Acta Endocrisol 1993: 129 (Supl): $27-30$.

6. Jacuet P: Medical therapy of protactinomas. Acta Endocrinol 1993; 129 (Supt): 31 -33.

7. Serri $O$ : Progress in the management of hyperprolactinemia. N Engl J Med 1994; 33 ]: 942-544.

8. Otverim $M$ da C. Abech D. Barbosa-Countimb $L$. Frereis $N$ : Macroprolactinema at 6 years of age: diagnostic dificulties, Arch Neuropsiquiatr 1992: 50 $397-401$.

9. Them D. Reggiardo D. Chates S, David R: Prolactinsecreting macroadenomas in adolescents: response to bromocriptine therapy. An J Dis Child 199, 147: 10.57.] 06 \mid$

10. Hemper $M$. Lee Vance $M$. Hon'ath E. Kowa's $K$ : The anterior pituitary. In Williams. Textbook of Endocrinology. Sth edit. W. Saunders Company, U.S.A. 1992:221-310.

11. Stck f. Friedmat E. Tadmor R. Sahar A, Karzneisom $D$ : Growth and puberty artest due to prolactinoma. Acta Paediatr Scand 1984: 73: 867-867.

12. Conserci $G$ : Prolactinoma in a prepubescen! girl. Am J Dis Child 1990: 144: 20-21.

13. Schwarte I. Hursey L. Grun J Howurd C: Prolactinoma associated with Iransient growth hormone deficiency but persistent growth retardation. J Pedials Endocrinol 1994: 7: 69-73.

14. Danzell G. Alkinson A. Carson D, Sheridan B: Nomal growith and pubertal development during bromocriptine treatment for a prolactin-secreting macroadenonia. Am J Dis Child 1986; ]40: 1216-1217.
15. Oberieid $S$, Nino $M$. Riddick $L$ et at: Combined bromocriptune growth hormone (GH) treatment in $\mathrm{GH}$ deficient children with macroprolaclinoma in sisu. J Clin Endocrinol Metab 1992; 75: 87-90.

16. Beck W. Stubbe $P$, Ludecke D: Prolactin producing pituitary adenoma in a 9 year old boy. Eur J Pediatr 1979: 130: 19.3-198.

17. Pallon M. Woolf P: Hyperprolactinemia and delayed puberty: A report of three cases and their response to therapy. Pediatrics 1983; 71: 572-575.

18. Blackwe/l $R$, Younger $J$ : Long rerm medical therapy and follow-up of pediatric-adolescent patients with prolactin-secreting macroadenoma. Fertil Steril 1986; 45: $713-716$.

19. Huseman C. Rizk G. Hohn F: Long-tem bromocriptine treattnent for prolactin-secreting macroadenoma. AIn J Dis Child 1986; 140: 12 16-1217.

20. Vun i Verial $J$ : The use of surgery for the treatment of prolactinomas. Acta Endocrinol 1993; 129 (Suppl. 1) 34-37

21. Katz E. Adashi E: Hyperprolactinemic disorders. Clin Obst Gynecol 1990: 33:622-639.

22. Molitch $M$ : Pregnancy and the hyperprolactinemic woman. N Engl J Med 1985; 312: 1364-1370.

23. Brue $T$. Pellegrini I. Priou A. Morange It Jayuet P: Prolactinomas atd resistance to dopamine agonists. Horm Res 1992; 38: 84-89

24. Pellegrini 1. Rashomjanahary $\boldsymbol{R}$. Gunz $G$, et al: Resistance to bromocriptine in prolactinomas. J Clin Endocrinol Metab 1989; 69: 500-509.

25. Lamberts $S$, Quit: $R$ : A comparison of the efficacy and safety of pergolide and bromocriptine in the treatment of hyperprolactinemia. J Clin Endocrinol Metab 1991; 72: 635-64l.

26. Brue $T$. Pellegrini $I$, Gunz $G$. ef at: Effects of the dopamine agonist CV 205-502 in human prolactinomas resistant to bromocriptine. J Clin Endocrinol Metab 1992: 74: $577-584$.

27. Von't Verlaat $J$. Croughs $R$, Brownell $J$ : Treatment of macroprolactinomas with a new non-ergot, long-act. ing dopaminergic drug, $\mathrm{CV}$ 205-502. Clin Endocrinol 1990: 33: 619.624.

28. Durantea L, Chanson P. Horlait S. Lubetzk J. Kuhn J: Effects of new dopaminergic agonisı CV 205-502 on plasma prolactin levels and tumor size in bromocriptinc-resistant prolactinomas. Clin Endocrinol 1991: 34: 25-29.

29. Lee Vance M. Lipper M. Klibanski A, Biller B. Stentar $N$, Molitich $M$ : Treatment of prolactinsecrcting piluitary, macroadenomas with long-acling non-ergot dopamine agonist CV 205-502. Alln Int Med 1990: I ]2: 668-673. 